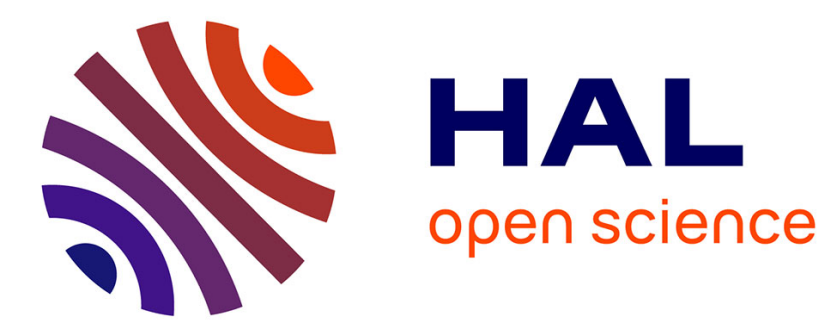

\title{
An LFR approach to varying sampling control of LPV systems: application to AUVs
}

Emilie Roche, Olivier Sename, Daniel Simon

\section{To cite this version:}

Emilie Roche, Olivier Sename, Daniel Simon. An LFR approach to varying sampling control of LPV systems: application to AUVs. ICSCS 2012 - 1st International Conference on Systems and Computer Science, Aug 2012, Villeneuve d'Ascq, France. hal-00746959

\section{HAL Id: hal-00746959 \\ https://hal.inria.fr/hal-00746959}

Submitted on 30 Oct 2012

HAL is a multi-disciplinary open access archive for the deposit and dissemination of scientific research documents, whether they are published or not. The documents may come from teaching and research institutions in France or abroad, or from public or private research centers.
L'archive ouverte pluridisciplinaire HAL, est destinée au dépôt et à la diffusion de documents scientifiques de niveau recherche, publiés ou non, émanant des établissements d'enseignement et de recherche français ou étrangers, des laboratoires publics ou privés. 


\section{An LFR approach to varying sampling control of LPV systems: application to AUVs}

\author{
Emilie Roche \\ Laboratoire IMS \\ 351 Cours de la Libération \\ 33405 Talence Cedex, France \\ emilie.roche@u-bordeaux1.fr
}

\author{
Olivier Sename \\ GIPSA-lab - Control Systems Dpt \\ $E N S E^{3}$ BP 46 \\ 38402 Saint Martin d'Hères Cedex, France \\ olivier.sename@gipsa-lab.fr
}

\author{
Daniel Simon \\ INRIA Grenoble Rhône-Alpes \\ Inovallée Montbonnot \\ 38334 Saint-Ismier Cedex, France \\ daniel.simon@inria.fr
}

\begin{abstract}
This paper deals with the varying sampling control of Linear Parameter Varying (LPV) systems, where varying sampling can be used to accommodate limited computation or sensory resources. A discrete-time Linear Fractional Representation of a LPV system is used to design a gain-scheduled controller, where both varying or uncertain parameters of the plant and the sampling rate are gathered in a unique varying parameters structure. The method is successfully applied for the case of the altitude control of an Autonomous Underwater Vehicle (AUV).
\end{abstract}

\section{INTRODUCTION}

In the context of network-controlled systems the idea of using varying control intervals naturally arises when the available computing power devoted to feedback control is limited, e.g. in embedded systems. It can be easily shown, e.g. [1], that decreasing the control frequency directly decreases the amount of computing needed for control. In that case a feedback scheduler is assumed to compute on-line new control intervals according to the CPU load and system's state. Another case is when sensing cannot be done at any time. For example, underwater vehicles mainly sense their environment using acoustic sensors. Due to the slow propagation of the acoustic signals, measurements are subject to delays increasing with the distance to the target. Also, to avoid cross-talking between acoustic sensors working in a narrow area these sensors must be scheduled, so that some of the sensors used in the control laws are triggered only at instants determined by an external manager [2]. In all these cases the control intervals are not equidistant, nevertheless they can be accurately measured by the local clock of the controller when the control computation is started.

Different control approaches have been considered to design varying sampling discrete-time controller. In [3] a gridding approach is used to design a discrete-time controller and observer with variable sampling. In [4] stability analysis of a controlled system under non uniform sampling is realized with a predictor/observer structure. Furthermore the authors have proposed in [5], [6] a LPV method for polytopic systems, under the assumption that the system representation is affine w.r.t the sampling period.

On the other hand Linear Parameter Varying representations are also increasingly used to deal with robust control of nonlinear systems. In particular this allows to represent some non- linearities as varying parameters (assuming some knowledge of internal measurements), and also to schedule a controller according to some performance criteria, e.g [7], [8].

The contribution stands here in the combination, for the first time, of these two ideas by synthesizing a discrete-time controller with a variable sampling rate for LPV systems. The key point then relies on the connection of the sampling parameter with the system's parameters. Indeed, when the number of varying parameters of the system increases, the inherent conservatism of the previously studied polytopic approach may lead to poor performances of the controlled system, or to unsolved problems due to numerical issues.

Another approach is proposed here to design sampling varying gain-scheduled controller for LPV systems, based on the Linear Fractional Representation (LFR). This method has already been studied in [9] concerning the synthesis of discrete-time gain-scheduled controller, depending only on the sampling period. This paper extends this previous work and deals with the design of a gain scheduled LFR controller w.r.t the sampling interval and w.r.t system's parameters, given a discrete-time Linear Fractional Representation (LFR) of the LPV varying sampling model.

The approach is then applied to an Autonomous Underwater Vehicle (AUV) for which for the operating constraints and plant's non-linearities fits well the LPV methodology.

In the next section, the LFR approach is presented, and an approach to get a discrete-time LPV model scheduled by both the plant's varying parameters and the varying sampling rate is described. In section III, two LPV models under LFR are developed, considering a hierarchical control structure (altitude and pitch angle control) for an Autonomous Underwater Vehicle (AUV). Finally in section IV, the two controllers of the hierarchical structure are designed using the LFR methodology with variable sampling period, and applied for the altitude control of the AUV.

\section{LFR APPROACH}

The LFR formulation is widely used in robust analysis to study the influence of the plant's uncertain parameters on the stability and performances of a closed-loop system. It can also be used to build a parameter dependent model of a dynamical system, depending on a known set of parameters. 
In particular, this approach can be used to keep some system's non linearities in a LFR model, and then linear control tools can be used to compute a controller scheduled by the parameters (as in[10]). In this section, the LFR formulation is presented and applied to the case of discrete-time sampling varying modeling and control of LPV systems.

\section{A. LFR model depending on system parameters}

The approach comes from the robust control theory and consists in separating the LTI part $P$ (not depending on the set of parameters) from the varying part $\Delta$ (parameters or uncertainties), as shown on figure 1.

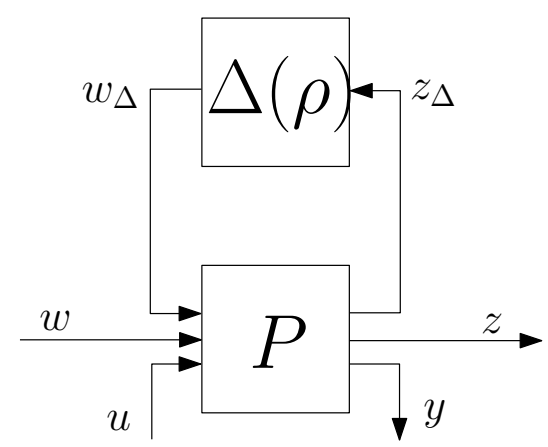

Fig. 1. System under LFR form

The matrix $\Delta$ represents the influence of the set of parameters $\rho($.$) on the plant. \rho($.$) is a varying parameter vector that$ takes values in the parameter space $\mathcal{P}_{\rho}$ such that:

$$
\begin{gathered}
\mathcal{P}_{\rho}:=\left\{\rho(.):=\left[\begin{array}{lll}
\rho_{1}(.) & \ldots & \rho_{n}(.)
\end{array}\right]^{T} \in \mathcal{R}^{N}\right. \\
\left.\rho_{i}(.) \in\left[\begin{array}{ll}
\underline{\rho}_{i} & \bar{\rho}_{i}
\end{array}\right] \forall i=1, \ldots, N\right\}
\end{gathered}
$$

where $N$ is the number of varying parameters. $\mathcal{P}_{\rho}$ is a convex set.

Here the vector of parameters $\rho($.$) represents plant's param-$ eters to be kept in the model, for example some non-linearities as in [11]. Note that the dependence of the system matrices w.r.t the parameters is not bounded to be affine, e.g. it can be rational.

For a LPV system depending on a vector of parameters $\rho($.$) an equivalent Linear Fractional Representation can be$ found as presented in Figure 1, with $P$ a continuous-time LTI plant $(P(s))$ and $\Delta$ a block diagonal matrix composed from the uncertain parameters.

The LFR representation is written as :

$$
\left[\begin{array}{c}
\dot{x} \\
\hline z_{\Delta} \\
z \\
y
\end{array}\right]=\left[\begin{array}{c|c}
\mathbf{A} & \mathbf{B} \\
\hline \mathbf{C} & \mathbf{D}
\end{array}\right]\left[\begin{array}{c}
x \\
\hline w_{\Delta} \\
w \\
u
\end{array}\right]
$$

where the continuous uncertain system's matrices as :

$$
\begin{array}{lll}
\mathbf{A} \text { (continuous plant state) } & \mathbf{B}=\left[B_{\Delta} B_{1} B_{2}\right] \\
\mathbf{C}=\left[\begin{array}{l}
C_{\Delta} \\
C_{1} \\
C_{2}
\end{array}\right] & \mathbf{D}=\left[\begin{array}{lll}
D_{\Delta \Delta} & D_{\Delta 1} & D_{\Delta 2} \\
D_{1 \Delta} & D_{11} & D_{12} \\
D_{2 \Delta} & D_{21} & D_{22}
\end{array}\right]
\end{array}
$$

From this model, a gain scheduled controller can be computed, depending on the same set of parameters $\Delta$ (or on a subset of $\Delta$ ) as presented in [12]. Here the LFR approach proposed in [9] will be extended to set-up a LFR model that accounts for system and sampling parameters. The steps below describe the proposed methodology.

\section{B. Parametrized discretization}

The interest of the previous LFR formulation is that the varying part is separated from the system dynamics. Then the LFR model in figure 1 can be discretized, assuming that the uncertain input/outputs $\left(w_{\Delta}\right.$ and $\left.z_{\Delta}\right)$ are sampled and hold at the sampling frequency. Then, in that context, the methodology proposed in [9] can be extended to get a sampling dependent LFR discrete-time model of an LPV system.

From the LFR model (1), where non-linearities are considered as varying parameters, a new LFR is computed by adding the sampling interval to the existing set of varying parameters.

The exact discretization w.r.t. a sampling interval $h$ of the LTI system $(\mathbf{A}, \mathbf{B}, \mathbf{C}, \mathbf{D})$ is given in equation (3)

$$
\mathcal{G}:\left\{\begin{aligned}
x_{k+1} & =A_{d}(h) x_{k}+B_{d}(h)\left[\begin{array}{lll}
w_{\Delta} & w & u
\end{array}\right]^{T} \\
{\left[\begin{array}{lll}
z_{\Delta} & z & y
\end{array}\right]^{T} } & =C x_{k}+D\left[\begin{array}{lll}
w_{\Delta} & w & u
\end{array}\right]^{T}
\end{aligned}\right.
$$

with:

$$
A_{d}(h)=e^{\mathbf{A} h} \quad B_{d}(h)=\int_{0}^{h} e^{\mathbf{A} \tau} d \tau \mathbf{B}
$$

The usual numerical method uses the matrix exponential :

$$
\left(\begin{array}{cc}
A_{d}(h) & B_{d}(h) \\
0 & I
\end{array}\right)=\exp \left(\left(\begin{array}{cc}
\mathbf{A} & \mathbf{B} \\
0 & 0
\end{array}\right) h\right)
$$

The sampling interval is assumed to be in $\left[h_{\text {min }}, h_{\text {max }}\right]$ with $h_{\min }>0$, it is evaluated around its nominal value $h_{0}$ as:

$$
h=h_{0}+\delta \quad \text { with } \quad h_{\min }-h_{0} \leq \delta \leq h_{\max }-h_{0}
$$

As explained in [5] equation (5) in this case becomes:

$$
\left(\begin{array}{cc}
A_{d}(h) & B_{d}(h) \\
0 & I
\end{array}\right)=\left(\begin{array}{cc}
A_{d}\left(h_{0}\right) & B_{d}\left(h_{0}\right) \\
0 & I
\end{array}\right)\left(\begin{array}{cc}
A_{d}(\delta) & B_{d}(\delta) \\
0 & I
\end{array}\right)
$$

with matrices $A_{h_{0}}=A_{d}\left(h_{0}\right), A_{\delta}=A_{d}(\delta), B_{h_{0}}=B_{d}\left(h_{0}\right)$ and $B_{\delta}=B_{d}(\delta)$ in equation 7 are defined following equation (5).

Matrices $A_{d}(h)$ and $B_{d}(h)$ depend both on the constant part $h_{0}$ and on the varying part $\delta$ of the sampling interval :

$$
\begin{aligned}
& A_{d}(h)=A_{h_{0}} A_{\delta} \\
& B_{d}(h)=B_{h_{0}}+A_{h_{0}} B_{\delta}
\end{aligned}
$$

Only the matrices $A_{\delta}$ and $B_{\delta}$ depend on the varying part of the sampling interval $\delta$ which appears in the varying part of the LFR model. An approximate form of equation (4) can be obtained by a Taylor series expansion at order $l$ (with a very good approximation for small values of $\delta$, as shown in [5]).

$$
\begin{aligned}
& A_{\delta}=I+\sum_{i=1}^{l} \frac{\mathbf{A}^{i}}{i !} \delta^{i}+o\left(\delta^{l+1}\right) \\
& B_{\delta}=\sum_{i=1}^{l} \frac{\mathbf{A}^{i-1} \mathbf{B}}{i !} \delta^{i}+o\left(\delta^{l+1}\right)
\end{aligned}
$$


The Taylor series expansion associated with the uncertain model leads to an LFR representation depending on the variation of the sampling interval $(\delta)$ and the plant's uncertain parameters $(\Delta)$ (see [9] for the details) as:

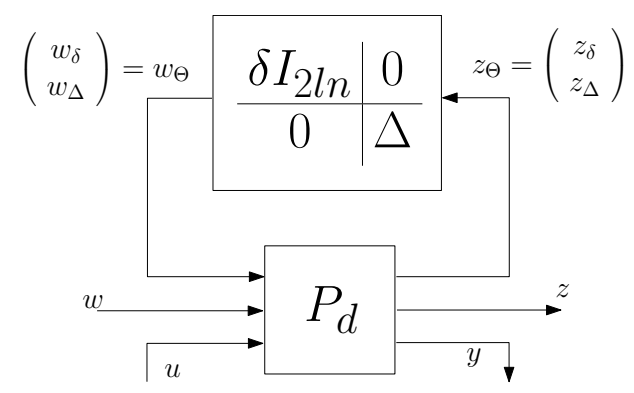

Fig. 2. LFR system depending on system parameters and sampling period variation

$$
P_{d}:\left\{\begin{array}{r}
x_{k+1}=\mathcal{A} x_{k}+\mathcal{B}\left[\begin{array}{c}
w_{\delta} \\
w_{\Delta} \\
w \\
u
\end{array}\right]_{k} \\
{\left[\begin{array}{c}
z_{\delta} \\
z_{\Delta} \\
z \\
y
\end{array}\right]_{k}=C x_{k}+\mathcal{D}\left[\begin{array}{c}
w_{\delta} \\
w_{\Delta} \\
w \\
u
\end{array}\right]_{k}}
\end{array}\right.
$$

with

$$
\begin{aligned}
& \Theta=\left[\begin{array}{cc}
\delta I_{2 \times l \times n} & 0 \\
0 & \Delta
\end{array}\right] \\
& \mathcal{A}=A_{h_{0}} \quad \mathcal{B}=\left(\begin{array}{lll}
\mathcal{B}_{1} & \mathcal{B}_{2} & B_{h_{0}}
\end{array}\right) \\
& \mathcal{C}=\left(\begin{array}{c}
C_{\delta} \\
\mathbf{C}
\end{array}\right) \quad \mathcal{D}=\left(\begin{array}{ccc}
\overline{\mathcal{D}} & 0 & 0 \\
0 & \overline{\mathcal{D}} & \mathcal{D}_{2 u} \\
0 & 0 & \mathbf{D}
\end{array}\right) \\
& \mathcal{B}_{1}=(A_{h_{0}} \mathbf{A} \underbrace{0_{n} \ldots 0_{n}}_{(1-1) \text { times }}) \quad \mathcal{B}_{2}=(A_{h_{0}} \underbrace{0_{n} \ldots 0_{n}}_{(1-1) \text { times }}) \\
& \mathcal{C}_{\delta}=(\underbrace{I_{n} \ldots I_{n}}_{1 \text { times }} \underbrace{0_{n} \ldots 0_{n}}_{1 \text { times }})^{T} \quad \overline{\mathcal{D}}=\left(\begin{array}{cccc}
0_{n} & \frac{\mathbf{A}}{2} & \ldots & 0_{n} \\
\vdots & \ddots & \ddots & \vdots \\
\vdots & \ddots & \ddots & \frac{\mathbf{A}}{l} \\
0_{n} & \ldots & \ldots & 0_{n}
\end{array}\right) \quad \mathcal{D}_{2 u}=\left(\begin{array}{c}
\mathbf{B} \\
\vdots \\
\mathbf{B}
\end{array}\right)
\end{aligned}
$$

where $l$ is the order of the Taylor series expansion order in $\delta$ and $n$ is the number of states of the continuous plant.

\section{LFR controller}

Following [12] an $H_{\infty}$ gain scheduled LFR controller can be computed from this discrete-time LFR model. This controller depends on the same set of varying parameters $\Theta$ (or on a subset of this one). The controller will be computed using tools derived from the bounded Real Lemma. Some performance specification are therefore fixed using weighting functions for loop shaping and $\mathcal{H}_{\infty}$ design. The dependence between the discrete-time LTI plant $P_{d}$, the controller $K$, the parameter block (including the system and sampling parameters) and the weighting functions $W_{i}$ and $W_{o}$ is depicted in Figure 3. Finally, the LFR controller is computed using the methodology presented in [12].

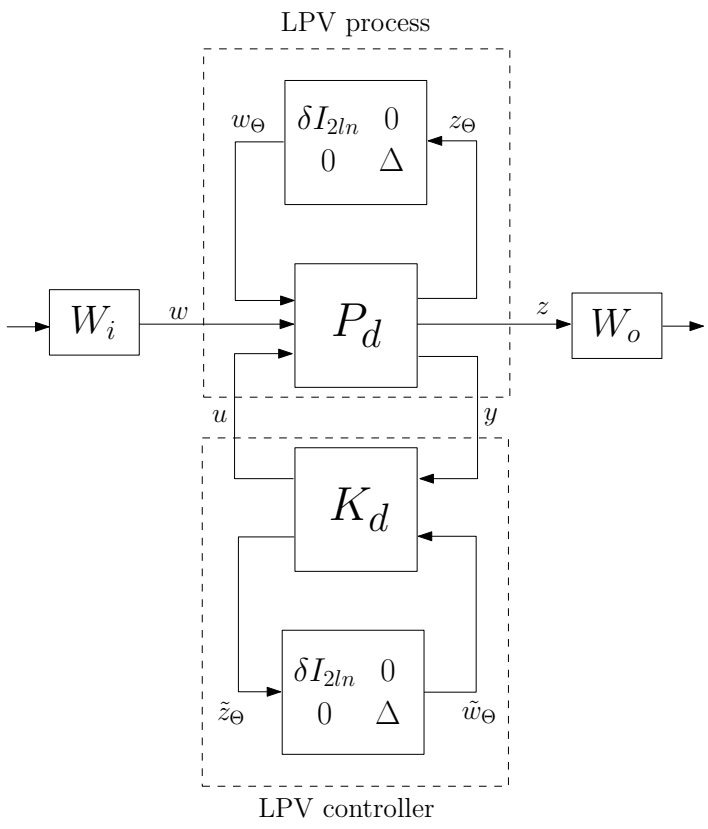

Fig. 3. LFR control structure

Note that the controller synthesis relies on the resolution of LMIs (as for the Polytopic case, see [6]). But conversely with the polytopic case, for the LFR case the number of LMIs to be solved do not depend on the number of parameters, only the sizes of the matrices in the LMIs are increasing with the size of the $\Theta$ matrix. It is worth noting that to get the solution of the control problems the YALMIP parser [13] and SeDuMi solver [14] have been used.

\section{A LPV DISCRETE-TIME MODEL OF THE AUV WITH SAMPLING DEPENDENCE}

The LFR formulation developed in the previous section is applied to the altitude control of an Autonomous Underwater Vehicle (AUV) using the hierarchical structure already used in [6], depicted in Figure 4. Two cascaded loops are used for altitude control, $\left(K_{z}(\delta)\right.$ and $\left.K_{\theta}(\delta, \rho)\right)$ based on two LPV models $\left(G_{d_{z}}(\delta)\right.$ and $\left.G_{d_{\theta}}(\delta, \rho)\right)$ detailed in the sequel.

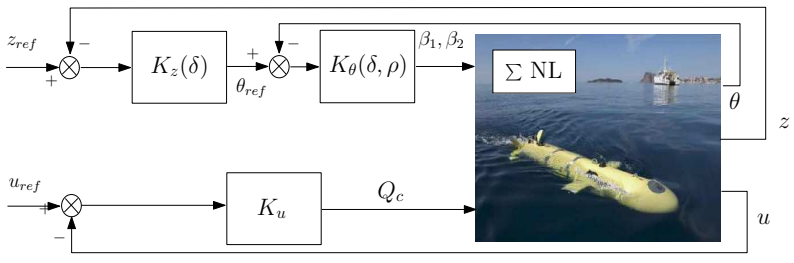

Fig. 4. Global control structure

The description of the full vehicle's dynamics uses a 12 dimensional state vector: 6 for the positions $x, y, z$ (linear 
positions) and $\phi, \theta$ and $\psi$ (roll, pitch an yaw angles) expressed in a fixed frame and 6 for the velocities (derivatives of the positions), $u, v, w, p, q, r$ expressed in the body frame.

\section{A. LPV model for the pitch angle}

To control motions around the pitch axis, a model reduction is considered keeping only the 2 state variables of interest, the pitch angle $\theta$ and the corresponding velocity $q$. The non-linear state equations corresponding to these states are :

$$
\begin{aligned}
\dot{\theta}= & \cos (\phi) q-\sin (\phi) r \\
M_{55} \dot{q}= & -\operatorname{pr}\left(I_{x}-I_{z}\right)-m\left[Z_{g}(q w-r v)\right] \\
& -\left(Z_{q} m-Z_{f} \mu V\right) g \sin (\theta) \\
& -\left(X_{q} m-X_{f} \mu V\right) g \cos (\theta) \cos (\phi) \\
& +M_{w q} w|q|+M_{q q} q|q|+\Gamma_{\theta}
\end{aligned}
$$

where $M_{55}$ is a diagonal element of the mass matrix (including water-added mass), $m$ is the mass of the vehicle, $V$ the volume of the hull and $\mu$ the density of the fluid. $I_{x}, I_{z}, Z_{g}, Z_{q}, Z_{f}, X_{q}, X_{f}, M_{w q}$ are inertial and hydrodynamics scalar coefficients. $\Gamma_{\theta}$ is the torque induced by the lift and drag actions on the control surfaces.

An LFR representation of this model is obtained using a tangential linearization around a variable equilibrium point : all position and speeds are taken equal to zero except the longitudinal speed $\left(u_{e q}\right)$ and the pitch angle $\left(\theta_{e q}\right.$ kept as a variable equilibrium point). The equations of the linearized model are :

$$
G_{\theta}:\left\{\begin{aligned}
\dot{\tilde{\theta}} & =q \\
M_{55} \dot{q} & =\left[-\left(Z_{g} m-Z_{f} \mu V\right) g \cos \left(\theta_{e q}\right)\right. \\
& \left.+\left(X_{g} m-X_{f} \mu V\right) g \sin \left(\theta_{e q}\right)\right] \tilde{\theta}+\Gamma_{\theta}
\end{aligned}\right.
$$

with $\tilde{\theta}=\theta-\theta_{e q}$.

The LFR formulation is used here to keep $\theta$ as a varying parameters into the model formulation. Indeed, in previous works, the limits of a simple linearization around a fixed equilibrium point appeared : when the pitch angle significantly deviates from 0 (the value chosen for linearization), the linearized model becomes too different from the actual one (due to the hydro-static return torque needing high actuators solicitations to keep the equilibrium around $\theta_{e q}$ ), leading to poor control performances.

An LFR model of the vehicle described in the form of figure 1 is derived from equations (19). The $\Delta$ block contains the varying part of the model, which depends on the linearization point $\left(\theta_{e q}\right)$. The LFR form is defined by :

$$
G_{\theta}:\left\{\begin{array}{r}
\left(\begin{array}{c}
\dot{\tilde{\theta}} \\
\dot{q}
\end{array}\right)=\mathcal{A}\left(\begin{array}{c}
\tilde{\theta} \\
q
\end{array}\right)+\left[\begin{array}{ll}
\mathcal{B}_{\Delta} & \mathcal{B}_{1}
\end{array}\right]\left(\begin{array}{c}
w_{\Delta} \\
u
\end{array}\right) \\
\left(\begin{array}{c}
z_{\Delta} \\
z
\end{array}\right)=\left[\begin{array}{c}
C_{\Delta} \\
I_{2}
\end{array}\right] x_{k}+\left[\begin{array}{ll}
0 & 0 \\
0 & 0
\end{array}\right]\left(\begin{array}{c}
w_{\Delta} \\
u
\end{array}\right)
\end{array}\right.
$$

with

$$
\begin{aligned}
\mathcal{A}=\left[\begin{array}{ll}
0 & 1 \\
0 & 0
\end{array}\right], \mathcal{B}_{\Delta}=\left[\begin{array}{cc}
0 & 0 \\
-\left(Z_{q} m-Z_{f} \mu V\right) g & \left(X_{q} m-X_{f} \mu V\right) g
\end{array}\right] \\
\mathcal{B}_{1}=\left[\begin{array}{cc}
0 & 0 \\
B_{\beta_{1}} & B_{\beta_{2}}
\end{array}\right], \mathcal{C}_{\Delta}=\left[\begin{array}{ll}
1 & 0 \\
1 & 0
\end{array}\right]
\end{aligned}
$$

where the gains $B_{\beta_{1}}$ and $B_{\beta_{2}}$ depend on the geometry of the control surfaces and are both proportional to $u_{e q}^{2}$ (assumed constant), and the control vector is $u=\left(\beta_{1} \beta_{2}\right)^{T}$.

$$
z_{\Delta}=\left[\begin{array}{c}
\tilde{\theta} \\
\tilde{\theta}
\end{array}\right] ; \quad w_{\Delta}=\Delta z_{\Delta} ; \Delta=\left[\begin{array}{cc}
\rho_{1} & 0 \\
0 & \rho_{2}
\end{array}\right]
$$

with:

$$
\left\{\begin{array}{l}
\rho_{1}=\cos \left(\theta_{e q}\right) \\
\rho_{2}=\sin \left(\theta_{e q}\right)
\end{array}\right.
$$

This model is then discretized, and the sampling interval, considered as a varying parameter in the model, is added to the $\Delta$ parameter block (as in section II-B).

Finally, choosing a Taylor series expansion of order $l=1$, a discrete-time LFR is obtained (as in equations (12) to (17)), with the parameter block $\Theta$ as:

$$
\Theta=\left[\begin{array}{ccc}
\delta \times I_{4} & 0 & 0 \\
0 & \rho_{1} & 0 \\
0 & 0 & \rho_{2}
\end{array}\right]
$$

\section{B. LPV model for the altitude}

Using the geometric relation between the altitude variations and the pitch angle, it appears that the the pitch angle, combined with the forward velocity, is in some sense the "actuator" to be used to generate altitude variations with low drag. Note that the cutoff frequency for the pitch controller is chosen faster than the one for the altitude controller to decouple the two loops, as done classically for cascade loops. Therefore the altitude controller computes the pitch angle setpoint needed to follow the desired path.

The projection of the body velocities in the fixed frame leads to a simple model :

$$
\dot{z}=u_{e q} \sin \theta
$$

A first order development of the sinus function is valid for the moderate pitch angles which are feasible by the AUV, which provides the linear model and transfer function :

$$
\begin{aligned}
\dot{z} & \simeq u_{e q} \theta \\
G_{z}(p) & =\frac{z}{\theta}=\frac{u_{e q}}{p}
\end{aligned}
$$

Therefore, the inner loop composed by the non linear model and the pitch angle controller $G_{\theta}$ can be approximated by an integrator, leading to a low-order controller for which the discretization step is very simple. The model given in (25) can be discretized by :

$$
z_{k+1}=z_{k}+u_{e q} \cdot h \cdot \theta_{k}
$$

The sampling interval is still assumed to belong to $\left[h_{\min }, h_{\max }\right]$, so that the altitude discrete-time model is :

$$
G_{d_{z}}(\delta): z_{k+1}=z_{k}+\left(h_{0}+\delta\right) \cdot u_{e q} \cdot \theta_{k}
$$

Therefore, assuming a constant forward velocity $u_{e q}$, the altitude model is scheduled by the sampling interval only. 


\section{Control Design}

To control the altitude of the AUV at a constant forward velocity, three controllers are designed to implement the structure of Figure 4. An $\mathcal{H}_{\infty}$ controller with a constant sampling period of $0.1 s$ is used to regulate the forward speed $u$ around $u_{e q}$, its classical design is not detailed here.

Two controllers are synthesized in discrete time to control the altitude $z$. The altitude controller $K_{z}(\delta)$ (computed from the model $\left.G_{d_{z}}(\delta)\right)$ gives a desired pitch angle $\theta_{r e f}$, which is used by the pitch angle controller $K_{\theta}(\delta, \rho)$ (model $G_{d_{\theta}}(\delta, \rho)$ ) to compute the actions $\beta_{1}$ and $\beta 2$ applied to the AUV.

\section{A. $\mathcal{H}_{\infty}$ loop shaping}

The two controllers involved in the altitude control loop are designed using the LFR methodology developed in II-C, based on $\mathcal{H}_{\infty}$ control design : some weighting functions are used to specify the performances of the closed loop system.

1) $K_{\theta}(\delta, \rho)$ : The pitch angle controller is designed using the structure of Figure 5. The weighting functions $W_{\theta_{e}}$ and

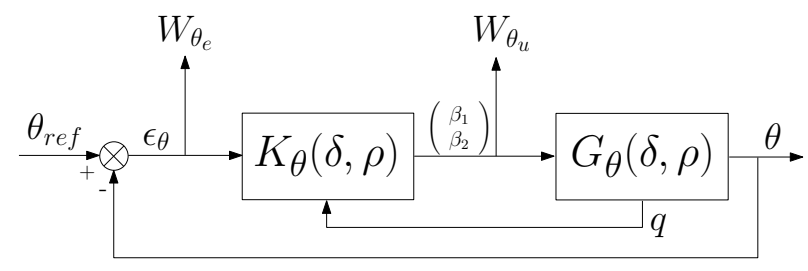

Fig. 5. Pitch control structure

$W_{\theta_{u}}$ are defined in discrete-time as follows:

$$
W_{\theta_{e}}=\frac{\frac{z}{M_{S_{\theta_{e}}}}+w_{S_{\theta_{e}}}}{z+w_{S_{\theta_{e}}} \varepsilon_{S_{\theta_{e}}}}, \quad W_{\theta_{u}}=\left[\begin{array}{cc}
0.8 & 0 \\
0 & 0.8
\end{array}\right]
$$

Remark: The weighting function on the tracking error is defined directly in discrete-time as a constant (LTI) first order filter. This correspond to an equivalent continuous time filter that depends on the period, allowing the adaptation of the performances with respect to the current sampling rate, as explained in [5].

The controller $K_{\theta}(\delta, \rho)$ is then computed as in [12] and its Bode diagram plotted in Figure 6 shows the adaptation of the controller gain w.r.t. the parameter vector. The pitch angle controller is scheduled by the "physical" parameters $\rho_{1}=\cos (\theta)$ and $\rho_{2}=\sin (\theta)$ and also the sampling interval $h$. The bounds on these parameters are chosen as follow: $\theta \in[-30 ; 30]$ so $\rho_{1} \in[0.86 ; 1]$ and $\rho_{2} \in[-0.5 ; 0.5]$ and the sampling interval $h \in[0.005 ; 0.03] s$.

2) $K_{z}(\delta)$ : The altitude controller is designed using a similar control configuration (see figure 7).

with weighting functions defined as follows:

$$
W_{z e}=\frac{\frac{z}{M_{S_{z e}}^{\prime}}+w_{S_{z e}}^{\prime}}{z+w_{S_{z e}}^{\prime} \varepsilon_{S_{z e}}^{\prime}}, \quad W_{z u}=5
$$

The controller is synthesized and its Bode diagram is presented in figure 8 , for 10 frozen value of the parameter $\delta$. The altitude controller is scheduled only by the sampling interval,varying inside the interval $[0.05 ; 0.3] s$.

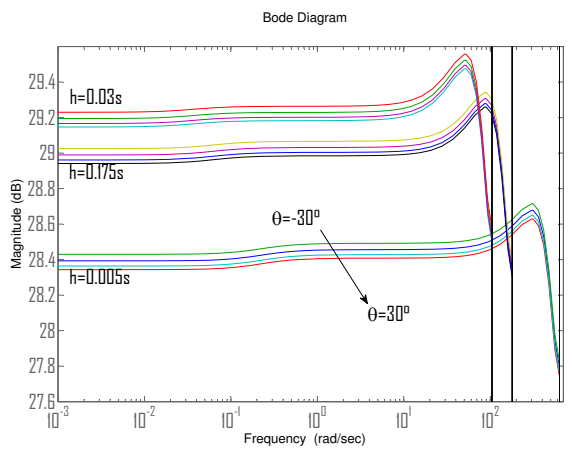

Fig. 6. Bode diagram of the pitch angle controller $K_{\theta}(\delta, \rho)$ for frozen values of the $\Theta_{e q}$ parameter

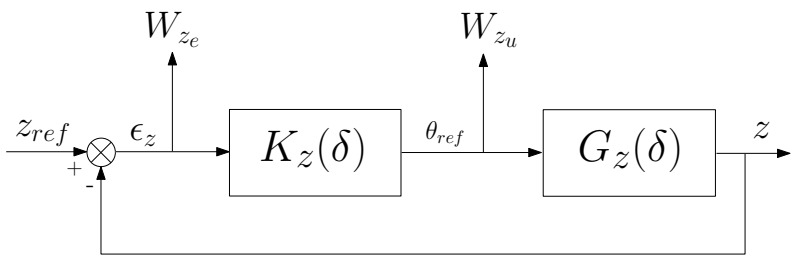

Fig. 7. Altitude control structure

\section{B. Simulation results}

The full (12 state variables) non-linear model (detailed in [9]) is used for the simulations, combined with the global control structure of Figure 4. The mission consists in bottom following at a constant altitude with a constant forward velocity (constant speed is needed for most payload, e.g., for map making using a sonar). An independent discrete-time $\mathcal{H}_{\infty}$ controller regulates the cruising speed $u$ around $1 \mathrm{~m} / \mathrm{s}$ during all the simulation.

For testing purpose the control interval $h$ is given as a sinusoidal wave (Figure 11 top right) and the step in the desired altitude is filtered to avoid unfeasible motion. The simulation results are given in Figures 9 to 11 . These results show the good adaptability of the controller to the variation of the parameters : whatever their variations, the closed-loop

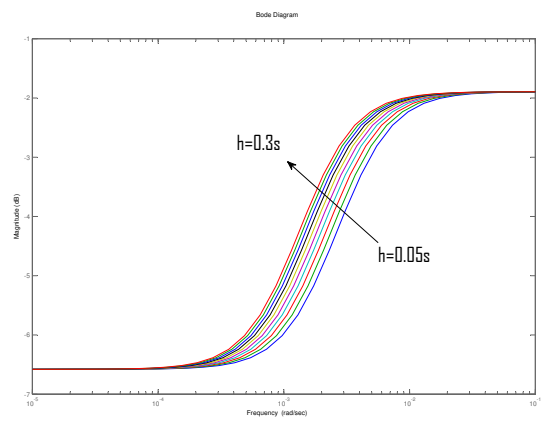

Fig. 8. Bode diagram of the altitude controller $K_{z}(\delta)$ 

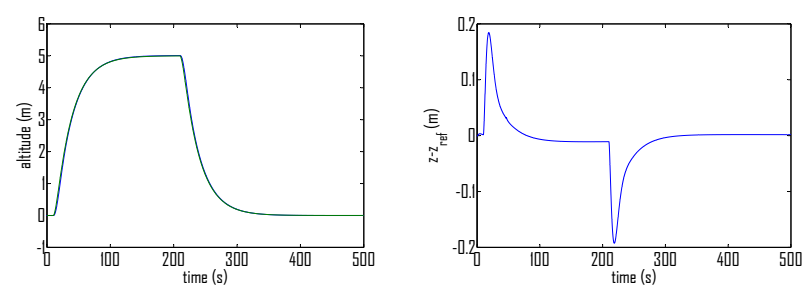

Fig. 9. Altitude reference and measure $z$ (left) altitude tracking error (right)
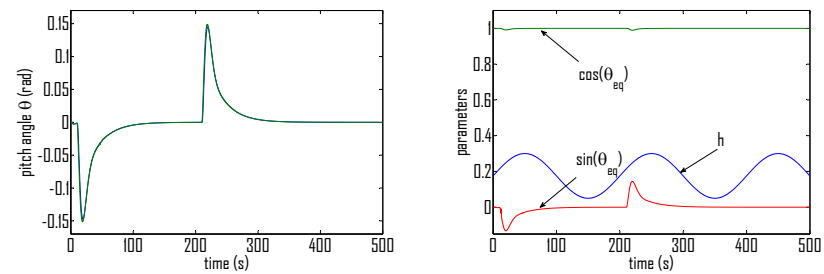

Fig. 10. Pitch reference and measure $\theta$ (left) Scheduling parameters (right)

stability is preserved for a large range of sampling intervals, and the tracking of the altitude and pitch angle references is achieved with a very good precision and time of response.

Compared with the same case study in [9], where only the sampling rate was considered as a varying parameter, considering here both the sampling interval and the plant's non-linearities $\left(\cos \left(\theta_{e q}\right)\right.$ and $\left.\sin \left(\theta_{e q}\right)\right)$ in the LPV model allows to improve the close loop performances, in particular through a more effective use of the actuators range. In the same case study, it was also observed that when using discrete-time $\mathcal{H}_{\infty}$ controllers designed for a constant sampling period, the control systems becomes unstable as soon as the sampling rate significantly deviates from the nominal value.

\section{CONCLUSiON}

In this paper, the problem of varying sampling control of an LPV system is addressed. An LFR methodology provides a unified framework to handle both the plant's non-linearities and the varying sampling rate in the same structure. The approach is applied on the example of the altitude control of a non-linear AUV.

Considering the sampling interval as a varying parameter of the LTI system makes the methodology well suited for the problem, since expressing the LPV system under a LFR makes
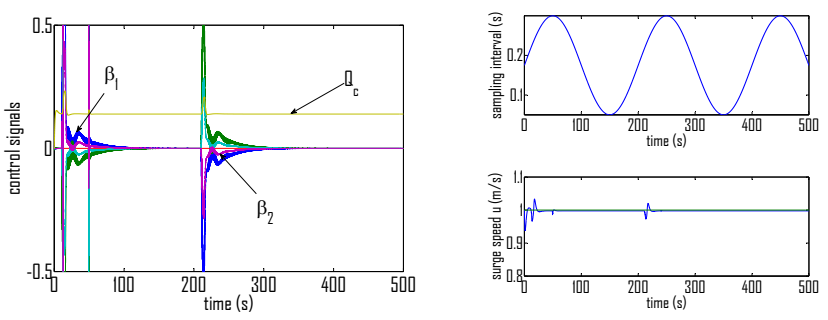

Fig. 11. left: Control signals ; right: Sampling interval (top) Speed (bottom) quite simple the discretization step. The loop-shaping weighting templates are also made sampling dependent to handle the closed-loop performance variations w.r.t. the actual sampling rate, and to preserve the stability margins. Furthermore the model is well suited for control synthesis through currently available LMI solvers.

However it is expected that these results, in particular their conservatism, might be improved considering improvements in LPV/LFR design, such as using new multipliers for Linear Fractional Transformations as in [15], or by using parameterdependent Lyapunov functions as in [16].

\section{REFERENCES}

[1] D. Simon, D. Robert, and O. Sename, "Robust control/scheduling codesign: application to robot control," in Real Time and Embedded Technology and Applications Symposium, 2005. RTAS 2005. 11th IEEE, march 2005, pp. $118-127$.

[2] J. Opderbecke, "Description of the scientific mission scenario(s) to be investigated for the marine application," FeedNetBack project, http://feednetback.eu/public-deliverables/public-deliverables-pdf/ ploneexfile.2009-12-09.1317343148/, deliverable D08.01, 2009.

[3] A. Sala, "Computer control under time-varying sampling period: An LMI gridding approach," Automatica, vol. 41, no. 12, pp. 2077-2082, 2005.

[4] A. Cuenca, P. J. Garcia Gil, K.-E. Arzén, and P. Albertos, "A predictorobserver for a networked control system with time-varying delays and non-uniform sampling," in Proceedings of the European Control Conference, Budapest, Aug 2009, Aug. 2009.

[5] D. Robert, O. Sename, and D. Simon, "An $\mathcal{H}_{\infty}$ LPV design for sampling varying controllers: experimentation with a t inverted pendulum," IEEE Transactions on Control Systems Technology, vol. 18, no. 3, pp. 741749, 2010.

[6] E. Roche, O. Sename, and D. Simon, "A hierarchical varying sampling $\mathcal{H}_{\infty}$ control for an AUV," in Proceedings of the IFAC World Congress, Milan, Italie, 2011.

[7] F. A. Shirazi, J. Mohammadpour Velni, and K. M. Grigoriadis, "An LPV design approach for voltage control of an electrostatic MEMS actuator," Microelectromechanical Systems, Journal of, vol. 20, no. 1, pp. $302-311$, feb. 2011.

[8] X. Wei and L. del Re, "Gain scheduled $\mathcal{H}_{\infty}$ control for air path systems of diesel engines using LPV techniques," Control Systems Technology, IEEE Transactions on, vol. 15, no. 3, pp. 406 -415, may 2007.

[9] E. Roche, O. Sename, and D. Simon, "LPV / $\mathcal{H}_{\infty}$ varying sampling control for autonomous underwater vehicles," in Proceedings of the IFAC SSSC, Ancona, Italie, 2010.

[10] C. Gauthier, O. Sename, L. Dugard, and G. Meissonnier, "An $\mathcal{H}_{\infty}$ linear parameter-varying (LPV) controller for a diesel engine common rail injection system," in Proceedings of the European Control Conference, Budapest, Hungary, 2007.

[11] J.-M. Biannic, A. Marcos, M. Jeanneau, and C. Roos, "Nonlinear simplified LFT modelling of an aircraft on ground," in IEEE International Conference on Control Applications, Munich, Germany, Oct. 2006, pp. $2213-2218$.

[12] P. Apkarian and P. Gahinet, "A convex characterisation of gain-scheduled $\mathcal{H}_{\infty}$ controllers," IEEE Transaction on Automatic Control, vol. 40, pp. 853 - 864, may 1995.

[13] J. Lofberg, "YALMIP : A toolbox for modeling and optimization in MATLAB," in Proceedings of the CACSD Conference, Taipei, Taiwan, 2004. [Online]. Available: http://control.ee.ethz.ch/ joloef/yalmip.php

[14] J. F. Sturm, "Using SeDuMi 1.02, a MATLAB toolbox for optimization over symmetric cones," Optim. Methods Softw., vol. 11/12, no. 1-4, pp. 625-653, 1999, interior point methods.

[15] C. W. Scherer, "LPV control and full block multipliers," Automatica, vol. 37, no. 3, pp. $361-375,2001$.

[16] F. Wu and K. Dong, "Gain-scheduling control of LFT systems using parameter-dependent Lyapunov functions," Automatica, vol. 42, no. 1, pp. $39-50,2006$. 